\title{
Effectiveness of Combined External Ventricular Drainage with Intraventricular Fibrinolysis for the Treatment of Intraventricular Haemorrhage with Acute Obstructive Hydrocephalus
}

\author{
Chinh Quoc Luong a, d Anh Dat Nguyen ${ }^{a, d}$ Chi Van Nguyen ${ }^{a, d}$ Ton Duy Mai ${ }^{a, d}$ \\ Tuan Anh Nguyen a, ${ }^{a}$ Son Ngoc Do ${ }^{a, d}$ Phuong Viet Dao ${ }^{a}$ Hanh Thi My Pham ${ }^{\text {h }}$ \\ Dung Thi Pham ${ }^{g}$ Hung Manh Ngok Quan Huu Nguyen ${ }^{\text {a,d }}$ Dat Tuan Nguyen ${ }^{a}$ \\ Thong Huu Tran a, ${ }^{a}$ Ky Van Le ${ }^{a}$ Nam Trong Do ${ }^{a} N_{g o c}$ Duc Ngo a, d \\ Vinh Duc Nguyen ${ }^{a}$ Hung Duc Ngo a,d Hai Bui Hoang ${ }^{a, f}$ Ha Viet Vu ${ }^{a}, f$ \\ Lan Tuong $\mathrm{Vu}^{\mathrm{a}} \mathrm{d}$ Binh Thanh Ngo ${ }^{i}$ Bai Xuan Nguyen ${ }^{j} \quad$ Dai Quoc Khuong ${ }^{a}$ \\ Dung Tien Nguyen $^{\mathrm{a}}$ Trung Xuan Vuong ${ }^{\mathrm{a}}$ Thu Hong Be ${ }^{c}$ Thomas Gaberel', $m$ \\ Lieu Van Nguyen b, e

\begin{abstract}
${ }^{a}$ Emergency Department, Bach Mai Hospital, Hanoi, Vietnam; bepartment of Neurology, Bach Mai Hospital, Hanoi, Vietnam; ' $P$ Poison Control Centre, Bach Mai Hospital, Hanoi, Vietnam; ${ }^{\mathrm{d}}$ Department of Emergency and Critical Care Medicine, Hanoi Medical University, Hanoi, Vietnam; e Department of Neurology, Hanoi Medical University, Hanoi, Vietnam; ${ }^{f}$ Emergency and Critical Care Department, Hanoi Medical University Hospital, Hanoi, Vietnam; ${ }^{9}$ Department of Science Management, Thai Binh University of Medicine and

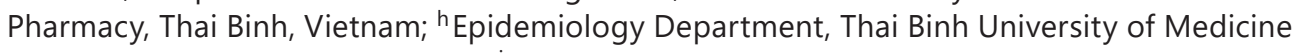
and Pharmacy, Thai Binh, Vietnam; 'Undergraduate Training and Management Department, Thai Binh University of Medicine and Pharmacy, Thai Binh, Vietnam; 'Department of Postgraduate Management, Thai Binh University of Medicine and Pharmacy, Thai Binh, Vietnam; ' Department of Neurosurgery II, Viet Duc Hospital, Hanoi, Vietnam; 'Department of Neurosurgery, Caen University Hospital, Caen, France; 'm Department of Neurosurgery, University of Caen Normandy, Caen, France
\end{abstract}

\section{Keywords}

Intraventricular haemorrhage $\cdot$ Intracerebral haemorrhage $\cdot$ External ventricular drainage Acute obstructive hydrocephalus - Intraventricular fibrinolysis · Recombinant tissue plasminogen activator

C.Q. Luong and A.D. Nguyen contributed equally as first authors.

Chinh Quoc Luong or Son Ngoc Do

Emergency Department, Bach Mai Hospital

No. 78, Giai Phong Road, Phuong Mai Ward

Dong Da District,Hanoi 100000 (Vietnam)

E-Mail luongquocchinh @gmail.com or sonngocdo@gmail.com
Hanh Thi My Pham

Epidemiology Department, Thai Binh University of Medicine and Pharmacy, No. 373, Ly Bon Street Thai Binh City, Thai Binh Province 410000 (Vietnam) E-Mail hanhhuongtb@yahoo.com 


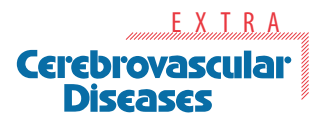

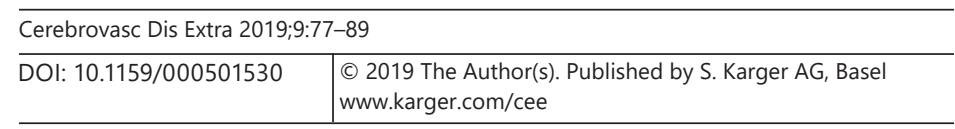

Luong et al.: Effectiveness of Combined EVD with IVF for the Treatment of IVH with $\mathrm{AOH}$

\section{Abstract}

Background: Intraventricular haemorrhage (IVH) patients with acute obstructive hydrocephalus $(\mathrm{AOH})$ who require external ventricular drainage (EVD) are at high risk for poor outcomes. Intraventricular fibrinolysis (IVF) with low-dose recombinant tissue plasminogen activator (rtPA) can be used to improve patient outcomes. Here, we evaluated the impact of IVF on the risk of death and the functional outcomes in IVH patients with AOH. Methods: This prospective cohort study included IVH patients with hypertensive intracranial haemorrhage complicated by $\mathrm{AOH}$ who required EVD. We evaluated the risk of death and the functional outcomes at 1 and 3 months, with a specific focus on the impact of combined EVD with IVF by low-dose rtPA. Results: Between November 30, 2011 and December 30, 2014, 80 patients were included. Forty-five patients were treated with EVD alone (EVD group) and 35 received IVF (EVD+IVF group). The 30- and 90-day mortality rates were lower in the EVD+IVF group than in the EVD group ( 42.2 vs. $11.4 \%, p=0.003$, and 62.2 vs. $20 \%, p<0.001$, respectively). The Graeb scores were significantly lower in the EVD+IVF group than in the EVD group $(p \leq 0.001)$ during the first 3 days and on day 7 after assignment. The 30-day good functional outcome (modified Rankin Scale [mRS] score 0-3) was also higher in the EVD+IVF group than in the EVD group (6.7 vs. $28.6 \%, p=0.008$ ). However, the 90 -day good functional outcome (mRS score $0-3$ ) did not significantly increase in the EVD+IVF group (30.8\% in the EVD group vs. $51.6 \%$ in the EVD+IVF group, $p=0.112$ ). Conclusions: In our prospective observational study, EVD+IVF was associated with a lower risk of death in IVH patients. EVD+IVF improved the chance of having a good functional outcome at 1 month; however, this result was no longer observed at 3 months.

(C) 2019 The Author(s)

Published by S. Karger AG, Basel

\section{Introduction}

Intraventricular haemorrhage (IVH) is a complication in $42 \%$ of intracerebral haemorrhage (ICH) cases and an independent risk factor for mortality and poor functional outcomes [1]. Acute obstructive hydrocephalus (AOH) following IVH potentially induces elevation of intracranial pressure (ICP), which increases the risk of death [2]. $\mathrm{AOH}$ can be controlled thanks to the insertion of an external ventricular drainage (EVD); however, the risk of death remains high. Injection of a fibrinolytic agent through the EVD, a treatment named intraventricular fibrinolysis (IVF), is known to facilitate removal of blood from the ventricles with a good safety profile $[3,4]$. In the recently published CLEAR III trial [4], IVF decreased the risk of death of patients with severe IVH but did not improve functional outcomes. However, some hope still exists concerning the use of IVF in IVH patients, especially for patients with the most severe IVH and for those with rapid clot removal. In the present cohort, we report our experience with IVH in patients who have severe IVH related to spontaneous ICH.

\section{Methods}

\section{Study Design and Sites}

This study was a prospective cohort study that included all patients with severe IVH requiring EVD. Our primary goal was to evaluate the impact of IVF with low-dose recombinant tissue plasminogen activator (rtPA). This study was purely observational, and no randomization was performed. The decision to inject rtPA was at the discretion of the physician in charge of the patients and the availability of the medication, which depended on the financial situation (either insurance or patient self-pay). Recruitment occurred between 
November 30, 2011 and December 30, 2014 in the Emergency Department of Bach Mai Hospital, Hanoi, Vietnam.

\section{Participants}

All patients had a clinical diagnosis of IVH with $\mathrm{AOH}$ related to a spontaneous supratentorial ICH that was not related to a macrovascular malformation and required EVD.

The inclusion criteria were as follows: patients aged 18-80 years with known symptom onset within $24 \mathrm{~h}$ of the initial computed tomography (CT) scan confirming IVH and 3rd or 4 th ventricle obstruction; a supratentorial ICH volume $\leq 30 \mathrm{~mL}$ as measured by the $\mathrm{ABC} / 2$ method; clot stability (no measured expansion $>5 \mathrm{~mL}$ ) on a repeat CT scan at least $6 \mathrm{~h}$ after EVD placement; systolic blood pressure $<200 \mathrm{~mm}$ Hg sustained for 6 hours; assignment to each group within $72 \mathrm{~h}$ of the CT scan diagnosing IVH; and a modified Rankin Scale (mRS) score of 0 or 1 before ICH.

The exclusion criteria were as follows: pregnancy; infratentorial ICH; suspicion or confirmation of a macroscopic vascular anomaly; ongoing coagulopathy; ongoing internal bleeding; multifocal, superficial bleeding observed at multiple vascular puncture and access sites or the site of a recent surgical intervention; a platelet count $<100,000 / \mu \mathrm{L}$; an international normalized ratio >1.4; and any other condition that would pose a significant hazard to the participants if the investigational therapy was initiated.

\section{Treatment and Procedures}

All patients were managed following the institutional protocol for the treatment of spontaneous ICH [5] as follows: early airway secured by endotracheal intubation if Glasgow Coma Scale (GCS) score $\leq 8$; proper sedation; mechanical ventilation; pharmacological treatment of cerebral oedema/mass effect (i.v. mannitol $20 \%$ or hypertonic saline $7.5 \%$ administration); and ICP monitoring. The cerebrospinal fluid (CSF) drainage system was kept open for drainage with a pressure level of $\geq 5 \mathrm{~cm} \mathrm{H}_{2} \mathrm{O}$; ICP was measured at least every $4 \mathrm{~h}$ with the CSF drainage system closed for at least $15 \mathrm{~min}$. The patient was treated if the ICP increased to $>20 \mathrm{~mm} \mathrm{Hg}$ for $>5$ min without any stimulation. In this case, standard emergency intensive care unit (ICU) measures for ICP control were taken, such as the following: (1) i.v. mannitol $20 \%$ or hypertonic saline $7.5 \%$ administration, proper sedation or hyperventilation only in mechanically ventilated patients; (2) if the ICP remained elevated after these measures, the protocol mandated early unclamping of the EVD and CSF drainage; and (3) if the ICP remained elevated, an emergent CT scan of the head was performed to exclude haemorrhagic complications. Blood pressure was monitored continuously using intra-arterial catheters and was treated when the mean arterial blood pressure exceeded $130 \mathrm{~mm} \mathrm{Hg}$.

The patients received either EVD alone (EVD group) or treatment with combined EVD and IVF by low-dose rtPA (Actilyse ${ }^{\circledR}$ ) (EVD+IVF group). A Camino ${ }^{\circledR}$ (Integra ${ }^{\mathrm{TM}}$, Plainsboro, NJ, USA) Ventricular Bolt catheter was placed in the frontal horn of the lateral ventricle with the least IVH bleeding. The proper EVD position was confirmed by a CT scan. Patients in both groups received the same ICU treatment according to an institutional protocol for ICH management [5]. Actilyse ${ }^{\circledR}$ was supplied by Boehringer Ingelheim (Germany). Patients received up to nine doses that were $8 \mathrm{~h}$ apart, and each dose was of $1 \mathrm{mg} / \mathrm{mL}$ of Actilyse ${ }^{\circledR}$.

Treatment continued for up to nine doses of alteplase unless the EVD was discontinued or an endpoint of clot lysis was reached (e.g., both the third and fourth ventricles were open, the IVH-related mass effect was resolved, or a Graeb score [6] of $\leq 4$ was reached); treatment was also discontinued if an adverse treatment endpoint occurred (e.g., symptomatic bleeding, infection, or death). The EVD was removed after $24 \mathrm{~h}$ of weaning. If the weaning failed, an internal shunt was inserted. 


\section{Variables}

Data were collected for each patient, including: age; sex; risk factors of intracranial haemorrhage; initial GCS score; mRS scores ranging from 0 (no disability) to 6 (death) [7-9]; Glasgow Outcome Scale (GOS) scores ranging from 1 (death) to 5 (good recovery) [10]; platelet count and international normalized ratio; ICH volume as calculated by the $\mathrm{ABC} / 2$ method; Evans ratio as measured by dividing the maximal width of the frontal horns by the maximal width of the inner table of the cranium at the level of the frontal horns; Graeb score [6]; ICH clot location; and times from symptom onset to hospital arrival, EVD placement, and group assignment. We also recorded ICP. Follow-up CT scans were performed daily to monitor asymptomatic bleeding and to measure clot resolution (indirectly assessed by the Graeb score) during the first 3 days. A CT/magnetic resonance imaging (MRI) scan was also obtained 1 week, 1 month, and 3 months after enrolment.

Bacterial ventriculitis was defined as a positive culture or, in the absence of a positive culture, $>50 \%$ polymorphonuclear leukocytes in the CSF count with a minimum of 50 cells counted or CSF glucose $<15 \mathrm{mg} / 100 \mathrm{~mL}$ ( $0.825 \mathrm{mmol} / \mathrm{L}$ ) [11]. Catheter occlusion was defined as non-patent EVD requiring irrigation.

Functional outcome assessment was performed by a certified examiner. The CT/MRI criterion used to define chronic hydrocephalus was marked symmetrical dilatation of the ventricles with an Evans ratio of at least 0.3 [12]. The participants had clinic visits on days 30 and 90 or phone contacts on day 90 after enrolment for the mRS and GOS assessments and evaluation of chronic hydrocephalus.

Data Analysis

Endpoints. The primary efficacy endpoint was mortality for all IVH patients with AOH requiring EVD. We also examined numerous secondary clinical endpoints (i.e., functional outcomes and complications).

Statistical Analyses. The data for each study patient were recorded from the same unified samples. The collected data were processed and analysed with $\mathrm{R}$ version 3.3 or higher ( $\mathrm{R}$ Foundation for Statistical Computing, Vienna, Austria). The differences in mortality between the two groups were tested with Pearson's $\chi^{2}$ algorithm. For the planned primary outcome analyses, we used the Kaplan-Meier time-to-event analysis to estimate the survival functions and the log-rank test to compare survival by treatment groups; Cox (proportional hazards) regression of association between mortality and potential determinants was also used, of which backward elimination was used to select variables. Linear regression was performed to find relationships between changes in the Graeb score and the functional outcomes (mRS). The values of the algorithms were considered statistically significant if the $p$ value was $<0.05$.

\section{Results}

\section{Patient Characteristics}

Eighty patients were included (Fig. 1), of whom 45 were treated with EVD alone (EVD group) and 35 with IVF (EVD+IVF group). Fifty-six patients (70\%) were men, the mean age was $57.1 \pm 12.6$ years, the mean GCS score was 8 (4-14), and the mean Graeb score was 9 (6-12). Then, we compared the patients in the EVD and EVD+IVF groups. All baseline variables shown in Table 1 were distributed evenly across the treatment groups. No significant difference between the EVD and EVD+IVF groups was found for the GCS score (7 [4-13] vs. 8 [5-14], respectively, $p=0.062$ ), systolic blood pressure (170.7 \pm 31.1 vs. $167.7 \pm 29.3 \mathrm{~mm} \mathrm{Hg}$, respectively, $p=0.667$ ), and the Graeb score (9 [6-12] vs. 8 [6-12], respectively, $p=0.863$ ) upon admission. 
Fig. 1. Flow chart of the study design and assessment occasions. CT, computed tomography; EVD, external ventricular drainage; GOS, Glasgow Outcome Scale; ICH, intracerebral haemorrhage; IVF, intraventricular fibrinolysis; IVH, intraventricular haemorrhage; MRI, magnetic resonance imaging; mRS, modified Rankin Scale.

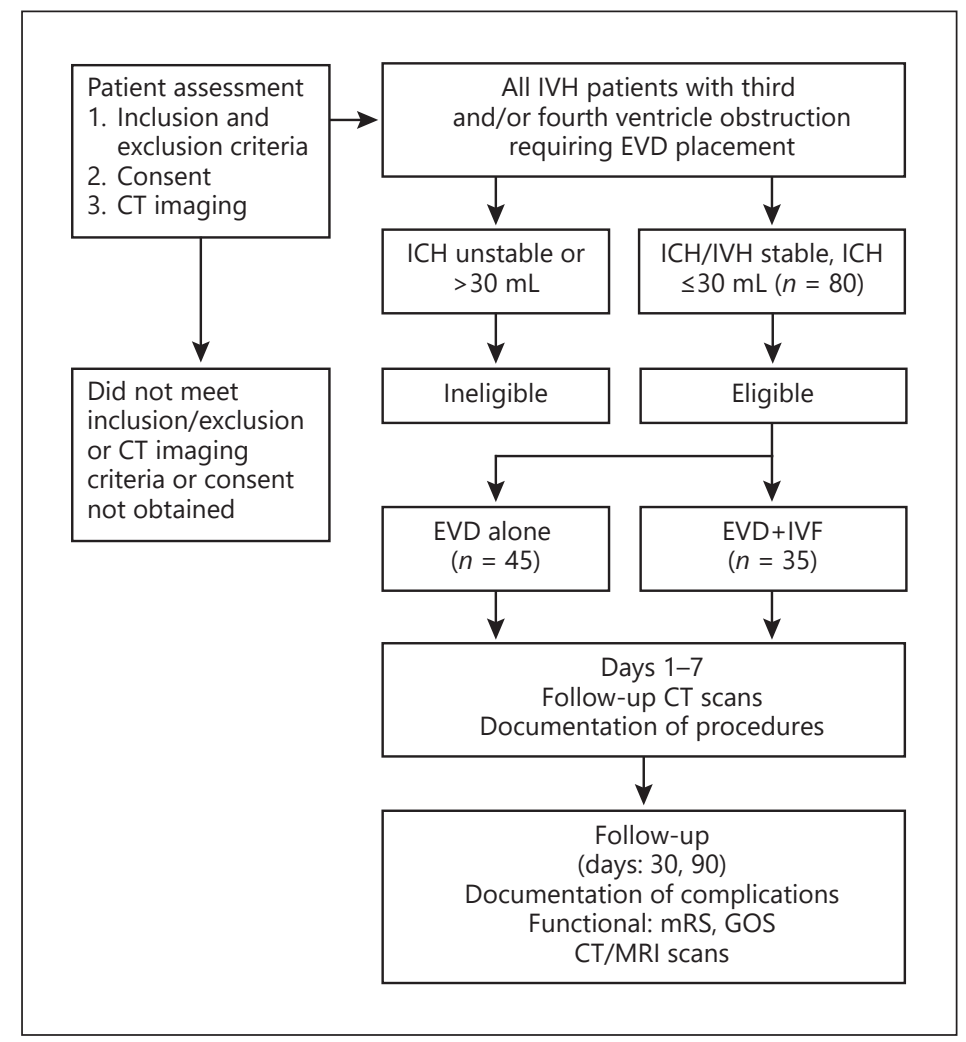

\section{Primary Endpoint}

Mortality. Twenty-three (28.75\%) patients died by the 30-day time point and 35 (43.75\%) patients died by the 90 -day time point. The 30- and 90-day mortality rates were higher in the EVD group than in the EVD+IVF group (19/45 [42.2\%] in the EVD group vs. $4 / 35$ [11.4\%] in the EVD+IVF group [ $p=0.003]$, and 28/45 [62.2\%] in the EVD group vs. $7 / 35$ [20\%] in the EVD+IVF group $[p<0.001]$, respectively) (Table 2). The estimated Kaplan-Meier survival probabilities were greater throughout the 90 days of follow-up for the EVD+IVF group than for the EVD group (cumulative case fatality: 28 [62.2\%] vs. 7 [20\%], $p<0.001$ ) (Fig. 2). Examination of the five potential determinants of mortality (baseline GCS score, baseline Graeb score, alteplase, first-day closed ICP, and initial ICH volume) revealed that only alteplase (defined as the number of $1 \mathrm{mg} / \mathrm{mL}$ alteplase doses) and first-day closed ICP (defined as the ICP measured after the CSF drainage system had been closed for at least $15 \mathrm{~min}$ on the first day after assignment) were significantly associated with mortality (Table 3 ). While each additional $1 \mathrm{mg}$ dose of alteplase was associated with a 29.1\% decrease in mortality risk (hazard ratio $=0.709$ [95\% confidence interval 0.536-0.938], $p=0.016$ ), each $1 \mathrm{~mm} \mathrm{Hg}$ increase in first-day closed ICP was associated with a 1.034-fold increase in mortality risk (hazard ratio $=1.034$ [95\% confidence interval 1.016-1.053], $p<0.001$ ). Though the effect size for firstday closed ICP was modest (hazard ratio $=1.034$ ), its association with mortality risk was highly significant $(p<0.001)$. A total of $26 / 80$ (32.5\%) patients were in grave condition or dying and were released to die at home, as requested by their family members. All of the patients who received "discharged to die" decisions were classified with an mRS score of 5 (severe disability) at the time of discharge; 17 of the patients died on the day of discharge or within 30 days of discharge (the other 8 died between 30 and 90 days of discharge), and a significant difference in "discharged to die" was found between the EVD and EVD+IVF groups 
Table 1. Baseline characteristics of patients treated with EVD+IVF or EVD alone

\begin{tabular}{|c|c|c|c|}
\hline Baseline variables & $\operatorname{EVD}(n=45)$ & EVD+IVF $(n=35)$ & $p$ value \\
\hline Age, years & $57.1 \pm 11.3$ & $57.1 \pm 14.3$ & 0.997 \\
\hline Male sex & $30(66.7 \%)$ & $26(74.3 \%)$ & 0.623 \\
\hline \multicolumn{4}{|l|}{ Health insurance status } \\
\hline Yes & $25(55.6 \%)$ & $23(65.7 \%)$ & 0.358 \\
\hline No & $20(44.4 \%)$ & $12(34.3 \%)$ & - \\
\hline \multicolumn{4}{|l|}{ Resident regions } \\
\hline Urban & $29(64.4 \%)$ & $22(62.9 \%)$ & 0.884 \\
\hline Rural & $16(35.6 \%)$ & $13(37.1 \%)$ & - \\
\hline \multicolumn{4}{|l|}{ Employment } \\
\hline Currently employed & $19(42.2 \%)$ & $15(42.9 \%)$ & 0.968 \\
\hline Retired & $20(44.4 \%)$ & $12(34.3 \%)$ & 0.579 \\
\hline Volunteer, student, unemployed & $4(8.9 \%)$ & $6(17.1 \%)$ & $0.502^{\mathrm{a}}$ \\
\hline \multicolumn{4}{|l|}{ Risk of intracranial haemorrhage } \\
\hline Stroke & $2(4.4 \%)$ & $3(8.6 \%)$ & $0.649^{a}$ \\
\hline Hypertension & $25(55.6 \%)$ & $26(74.3 \%)$ & 0.104 \\
\hline Diabetes & $7(15.6 \%)$ & $2(5.7 \%)$ & $0.286^{\mathrm{a}}$ \\
\hline Smoking & $4(8.9 \%)$ & $3(8.6 \%)$ & $1.000^{\mathrm{a}}$ \\
\hline Antiplatelet use & $1(2.2 \%)$ & 0 & - \\
\hline Alcohol abuse & $3(6.7 \%)$ & $2(5.7 \%)$ & - \\
\hline Seizures & $1(2.2 \%)$ & 0 & - \\
\hline Liver disease & $1(2.2 \%)$ & 0 & - \\
\hline Renal disease & $1(2.2 \%)$ & 0 & - \\
\hline \multicolumn{4}{|c|}{ Clinical and laboratory characteristics on admission } \\
\hline GCS & $7[4-13]$ & $8[5-14]$ & 0.062 \\
\hline Systolic BP, mm Hg & $170.7 \pm 31.1$ & $167.7 \pm 29.3$ & 0.667 \\
\hline Diastolic BP, mm Hg & $97.6 \pm 20.9$ & $97.4 \pm 15.8$ & 0.976 \\
\hline Hemiplegia & $34(75.6 \%)$ & $28(80.0 \%)$ & 0.637 \\
\hline Preadmission mRS score $=1$ & $2(4.4 \%)$ & $3(8.6 \%)$ & 0.649 \\
\hline Preadmission mRS score $=0$ & $43(95.6 \%)$ & $32(91.4 \%)$ & - \\
\hline Platelet count, $\times 10^{9} / \mathrm{L}$ & $247.9 \pm 86.2$ & $248.1 \pm 59.9$ & 0.992 \\
\hline International normalized ratio & $1.05 \pm 0.13$ & $1.01 \pm 0.1$ & 0.151 \\
\hline \multicolumn{4}{|l|}{ Imaging characteristics on admission } \\
\hline Graeb score & $9[6-12]$ & $8[6-12]$ & 0.863 \\
\hline Supratentorial ICH & $37(82.2 \%)$ & $26(74.3 \%)$ & 0.389 \\
\hline ICH volume, $\mathrm{mL}^{\mathrm{b}}$ & $13.9 \pm 7.2$ & $14.4 \pm 7.4$ & 0.799 \\
\hline Evans ratio ${ }^{c}$ & $0.42 \pm 0.06$ & $0.41 \pm 0.07$ & 0.649 \\
\hline \multicolumn{4}{|l|}{ ICH clot location } \\
\hline Central grey nuclei & $36(80 \%)$ & $26(74.3 \%)$ & 0.470 \\
\hline Internal capsular & $1(2.2 \%)$ & $1(2.9 \%)$ & $0.687^{\mathrm{a}}$ \\
\hline \multicolumn{4}{|l|}{ Others } \\
\hline Ictus to hospital arrival, $\mathrm{h}$ & $4[1-20]$ & $6[1-20]$ & 0.188 \\
\hline Ictus to EVD, $\mathrm{h}$ & $10[2-23]$ & $10[2-22]$ & 0.680 \\
\hline Ictus to IVF, $\mathrm{h}$ & - & $23[18-40]$ & - \\
\hline
\end{tabular}

Values are expressed as mean \pm standard deviation, $n(\%)$, or median [interquartile range]. Urban was defined as city, suburb, outskirts, or town. BP, blood pressure; EVD, external ventricular drainage; GCS, Glasgow Coma Scale; ICH, intracerebral haemorrhage; IVF, intraventricular fibrinolysis; mRS, modified Rankin Scale. ${ }^{\text {a }}$ Fisher's exact test (one-sided). ${ }^{\mathrm{b}}$ Calculated by the ABC/2 method. ${ }^{\mathrm{c}}$ Measured by dividing the maximal width of the frontal horns by the maximal width of the inner table of the cranium at the level of the frontal horns. 
Table 2. Comparison of outcomes between patients treated with EVD+IVF and EVD alone

\begin{tabular}{|c|c|c|c|}
\hline & $\operatorname{EVD}(n=45)$ & $\operatorname{EVD}+\operatorname{IVF}(n=35)$ & $p$ value \\
\hline \multicolumn{4}{|l|}{ Emergency ICU length of stay, days } \\
\hline All patients & $8[2-54]$ & $14[2-53]$ & 0.002 \\
\hline 30-day survival patients & $14[6-54]$ & 17 [9-53] & 0.058 \\
\hline \multicolumn{4}{|l|}{ EVD duration, days } \\
\hline All patients & $5[2-15]$ & $7[2-14]$ & 0.018 \\
\hline 30-day survival patients & $7[2-15]$ & $7[3-14]$ & 0.956 \\
\hline Tracheostomy & $14(31.1 \%)$ & $11(31.4 \%)$ & 0.976 \\
\hline \multicolumn{4}{|l|}{ CT/MRI scans } \\
\hline 30 days $(n=80)$ & $4(8.9 \%)$ & $20(57.1 \%)$ & $<0.001^{\mathrm{a}}$ \\
\hline 90 days $(n=57)$ & $6(23.1 \%)$ & $13(41.9 \%)$ & $0.422^{\mathrm{a}}$ \\
\hline Ventriculoperitoneal shunt & 0 & $2(5.7 \%)$ & $0.188^{\mathrm{a}}$ \\
\hline \multicolumn{4}{|c|}{ Complications related to EVD and/or IVF } \\
\hline Intracranial rebleeding & $5(11.1 \%)$ & $2(5.7 \%)$ & $0.328^{\mathrm{a}}$ \\
\hline Catheter tract bleeding & $1(2.2 \%)$ & $2(5.7 \%)$ & $0.406^{\mathrm{a}}$ \\
\hline Catheter occlusion & $8(17.8 \%)$ & $2(5.7 \%)$ & $0.099^{\mathrm{a}}$ \\
\hline Ventriculitis & $4(8.9 \%)$ & $3(8.6 \%)$ & $0.640^{\mathrm{a}}$ \\
\hline Chronic hydrocephalus & $3(6.7 \%)$ & $10(28.6 \%)$ & $0.036^{\mathrm{a}}$ \\
\hline Untreated chronic hydrocephalus & $3(6.7 \%)$ & $8(22.9 \%)$ & $0.107^{\mathrm{a}}$ \\
\hline \multicolumn{4}{|l|}{ General complications } \\
\hline Pneumonia & $9(20 \%)$ & $12(34.3 \%)$ & $0.150^{\mathrm{a}}$ \\
\hline Urinary tract infection & $3(6.7 \%)$ & $4(11.4 \%)$ & $0.360^{\mathrm{a}}$ \\
\hline \multicolumn{4}{|c|}{ Good functional outcomes (mRS score 0-3) } \\
\hline 30 days & $3(6.7 \%)$ & $10(28.6 \%)$ & 0.008 \\
\hline 90 days & $8(30.8 \%)$ & $16(51.6 \%)$ & 0.112 \\
\hline "Discharged to die" & $21(46.7 \%)$ & $5(14.3 \%)$ & 0.002 \\
\hline Within first 3 days of care & $14(31.1 \%)$ & 0 & - \\
\hline After first 3 days of care & $7(15.6 \%)$ & $5(14.3 \%)$ & 0.241 \\
\hline \multicolumn{4}{|l|}{ Mortality rate } \\
\hline 30 days & $19(42.2 \%)$ & $4(11.4 \%)$ & 0.003 \\
\hline 90 days & $28(62.2 \%)$ & $7(20 \%)$ & $<0.001$ \\
\hline
\end{tabular}

Values are expressed as $n(\%)$ or median [interquartile range]. Intracranial rebleeding was defined as combined intracerebral haemorrhage and intraventricular haemorrhage rebleeding; "discharged to die" defines patients who were in grave condition or dying and were released to die at home, as requested by their family members. CT, computed tomography; EVD, external ventricular drainage; ICU, intensive care unit; IVF, intraventricular fibrinolysis; MRI, magnetic resonance imaging; mRS, modified Rankin Scale. ${ }^{\text {a }}$ Fisher's exact test (one-sided).

(21/45 [46.7\%] vs. $5 / 35$ [14.3\%], respectively, $p<0.001)$ (Table 2). In the first 3 days of care, patients (14/45 [31.1\%]) who received "discharged to die" decisions were observed in the EVD group, while these decisions were found in both groups beyond the first 3 days of care (7/45 [15.6\%] in the EVD vs. 5/35 [14.3\%] in the EVD+IVF group, $p=0.241$ ) (Table 2).

\section{Secondary Endpoints}

Good Functional Outcomes. After 30 days, $3 / 45$ (6.7\%) patients in the EVD group had good functional outcome (mRS score $0-3$ ) versus 10/35 (28.6\%) patients in the EVD+IVF group ( $p=0.008$ ). No significant difference in 90 -day good functional outcome (mRS score 


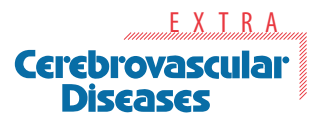

Fig. 2. Kaplan-Meier curve demonstrating survival in the treatment group of IVH patients with $\mathrm{AOH}$. The treatment groups were categorized as EVD alone (EVD group) or combined EVD with IVF by low-dose rtPA (EVD+IVF group). A significant difference in survival exists between the curves ( $p<0.001$ with the logrank test). The dashed line represents the EVD+IVF group and the solid line represents the EVD group. $\mathrm{AOH}$, acute obstructive hydrocephalus; EVD, external ventricular drainage; IVF, intraventricular fibrinolysis; IVH, intraventricular haemorrhage; rtPA, recombinant tissue plasminogen activator.

Fig. 3. Improvement of the Graeb score by treatment group during the first 3 days (throughout dosing) after assignment to each group. The Graeb scores were significantly lower in the EVD+IVF group than in the EVD group ${ }^{*} p \leq 0.001$ with the Mann-Whitney U test). Day 1 was the first day after assignment to each group. The dashed line represents the EVD+IVF group and the solid line represents the EVD group. EVD, external ventricular drainage; IVF, intraventricular fibrinolysis.

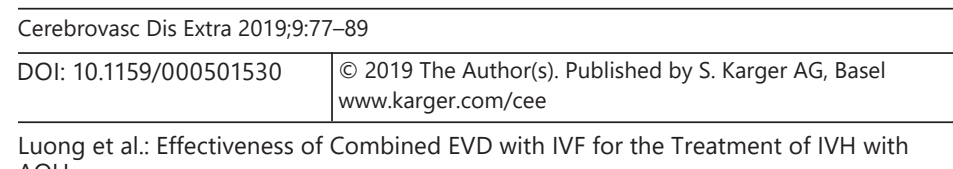

Luong et al.: Effectiveness of Combined EVD with IVF for the Treatment of IVH with $\mathrm{AOH}$
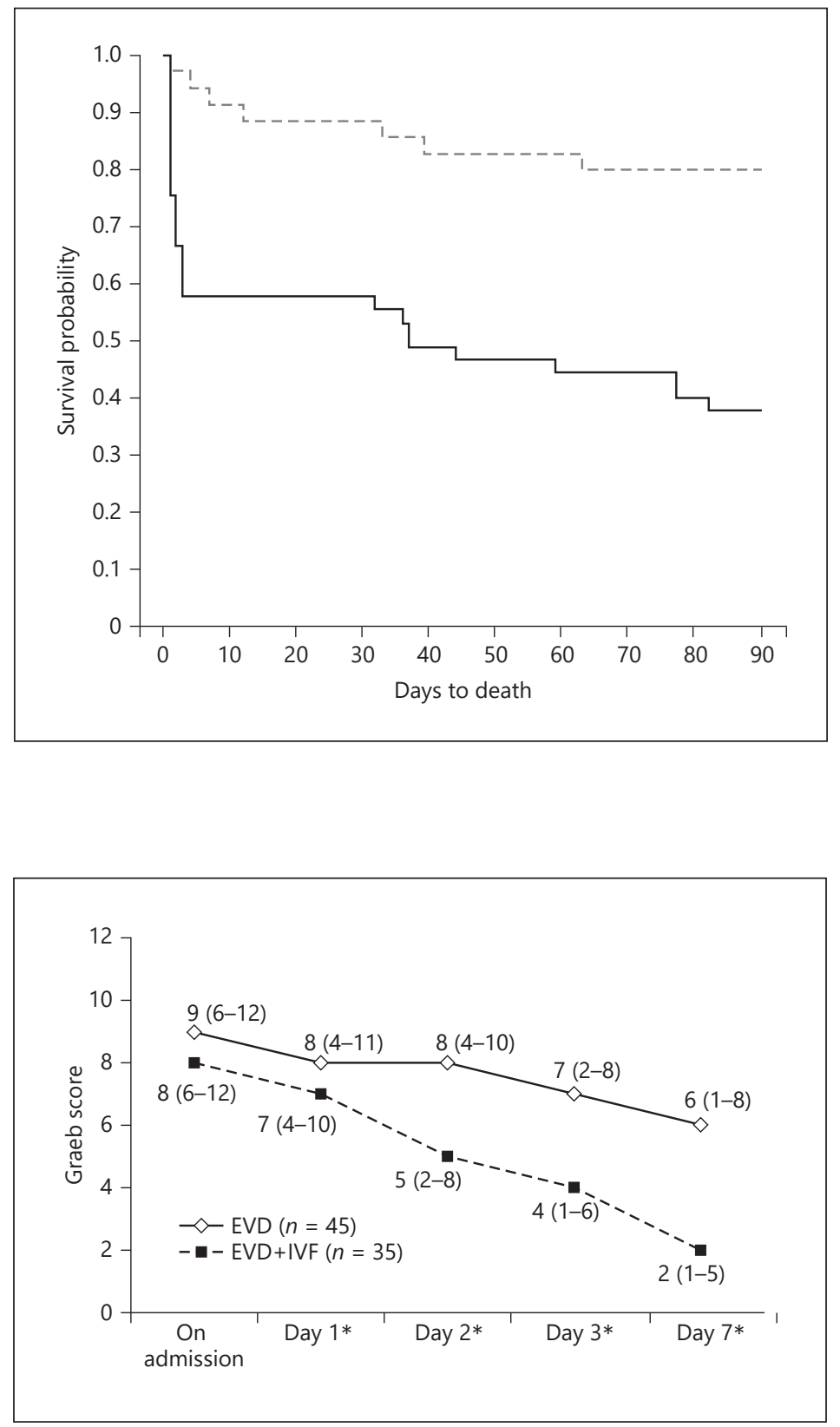

$0-3)$ was observed between the two groups (8/26 [30.8\%] in the EVD group vs. 16/31 [51.6\%] in the EVD+IVF group; $p=0.112$ ).

Clot Removal and Catheter Management. Ventricular blood was removed faster in the EVD+IVF group than in the EVD group ( $p \leq 0.001$ ) (Fig. 3). Examination of the relationship between the change in the Graeb score (defined as the difference between the two Graeb scores obtained at admission and on day 3 ) and the functional outcome (30-day mRS score) revealed that each 5-point increase in the change in the Graeb score was significantly associated with a 0.710 -point increase in the 30 -day mRS score (coefficient $0.710,95 \%$ confidence interval $0.137-1.283, p=0.016$ ); thus, less IVH clot removal may result in poorer functional outcome (online suppl. Table A20; for all online suppl. material, see www. karger.com/doi/10.1159/000501530). No significant difference was found in the time 
Table 3. Association between mortality and potential determinants: Cox regression analysis (backward elimination) (56 men and 24 women)

\begin{tabular}{|c|c|c|c|c|c|c|}
\hline \multirow[t]{2}{*}{ Steps } & \multirow[t]{2}{*}{ Potential determinants } & \multirow[t]{2}{*}{ Unit } & \multirow[t]{2}{*}{ HR } & \multicolumn{2}{|c|}{ 95\% CI for HR } & \multirow[t]{2}{*}{$p$ value } \\
\hline & & & & lower & upper & \\
\hline \multirow[t]{5}{*}{1} & Baseline GCS & 1 point & 0.856 & 0.712 & 1.029 & 0.098 \\
\hline & Baseline Graeb score & 1 point & 1.163 & 0.890 & 1.518 & 0.269 \\
\hline & Alteplase & $1 \mathrm{mg}$ & 0.697 & 0.528 & 0.921 & 0.011 \\
\hline & First-day closed ICP & $1 \mathrm{~mm} \mathrm{Hg}$ & 1.038 & 1.018 & 1.058 & $<0.001$ \\
\hline & Initial ICH volume & $1 \mathrm{~mL}$ & 1.024 & 0.975 & 1.074 & 0.346 \\
\hline \multirow[t]{4}{*}{2} & Baseline GCS & 1 point & 0.860 & 0.717 & 1.030 & 0.102 \\
\hline & Baseline Graeb score & 1 point & 1.147 & 0.878 & 1.499 & 0.316 \\
\hline & Alteplase & $1 \mathrm{mg}$ & 0.696 & 0.526 & 0.920 & 0.011 \\
\hline & First-day closed ICP & $1 \mathrm{~mm} \mathrm{Hg}$ & 1.037 & 1.018 & 1.057 & $<0.001$ \\
\hline \multirow[t]{3}{*}{3} & Baseline GCS & 1 point & 0.855 & 0.710 & 1.030 & 0.100 \\
\hline & Alteplase & $1 \mathrm{mg}$ & 0.709 & 0.536 & 0.938 & 0.016 \\
\hline & First-day closed ICP & $1 \mathrm{~mm} \mathrm{Hg}$ & 1.034 & 1.016 & 1.053 & $<0.001$ \\
\hline
\end{tabular}

Baseline GCS is the GCS on admission; baseline Graeb score is the Graeb score on admission; alteplase is the number of $1 \mathrm{mg} / \mathrm{mL}$ alteplase doses; first-day closed ICP was defined as the ICP measured while the cerebrospinal fluid drainage system was closed for at least $15 \mathrm{~min}$ on the first day after assignment (a patient could have up to 8 closed ICPs measured per day; in this study, we randomly selected only one from all closed ICPs for each patient); initial ICH volume is ICH volume on admission. CI, confidence interval; GCS, Glasgow Coma Scale; HR, hazard ratio; ICH, intracerebral haemorrhage; ICP, intracranial pressure.

from ictus to hospital arrival between the two groups (4 [1-20] h in the EVD group vs. 6 [1-20] $\mathrm{h}$ in the EVD+IVF group, $p=0.188$ ) (Table 1). The time from ictus to EVD placement was similar between the groups $(10$ [2-23] $\mathrm{h}$ in the EVD group vs. 10 [2-22] h in the EVD+IVF group, $p=0.680$ ) (Table 1 ). Most patients were assigned within $36 \mathrm{~h}$ of ictus (online suppl. Table A2), and the time from ictus to the first IVF dose among the patients receiving IVF was $23(18-40)$ h (Table 1). No patients were treated with bilateral EVD. EVD duration was significantly shorter in the EVD group (5 [2-15] days) than in the EVD+IVF group (7 [2-14] days) ( $p=0.018)$. However, no significant difference was found in the EVD duration among patients who survived 30 days between the two groups (7 [2-15] days in the EVD group vs. 7 [3-14] days in the EVD+IVF group, $p=0.956)$. The EVD group had a significantly shorter emergency ICU length of stay (8 [2-54] days) than the EVD+IVF group (14 [2-53] days) ( $p=0.002)$, but no significant difference was observed in the emergency ICU length of stay among the patients who survived 30 days between the two groups (14 [6-54] days in the EVD group vs. 17 [9-53] days in the EVD+IVF group, $p=0.058$ ) (Table 2). A significant difference was found in chronic hydrocephalus between the two groups (3/45 [6.7\%] patients in the EVD group vs. $10 / 35$ [28.6\%] patients in the EVD+IVF group, $p=0.036$ ) (Table 2).

Complications. Intracranial rebleeding and catheter tract bleeding were observed in 5/45 (11.1\%) and 1/45 (2.2\%) patients, respectively, in the EVD group, and both outcomes occurred in 2/35 (5.7\%) patients in the EVD+IVF group. Ventriculitis was diagnosed in 4/45 $(8.9 \%)$ patients in the EVD group and $3 / 35(8.6 \%)$ patients in the EVD+IVF group. Catheter occlusion was also observed in 8/45 (17.8\%) patients in the EVD group and 2/35 (5.7\%) patients in the EVD+IVF group. 


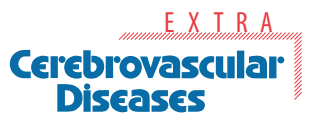

Fig. 4. Outcome of the mRS scores (scores range from 0 [no disability] to 6 [death]) at 30 and 90 days by treatment group. The dashed lines indicate the differences in proportion of 90-day mRS score $\leq 3$ (8 [30.8\%] in the EVD group vs. 16 [51.6\%] in the EVD+IVF group, $p=0.112$ ) and 90-day mRS score $=6(9[34.6 \%]$ in the EVD group vs. 3 [9.7\%] in the EVD+IVF group, $p=0.021$ ). EVD, external ventricular drainage; IVF, intraventricular fibrinolysis; mRS, modified Rankin Scale.

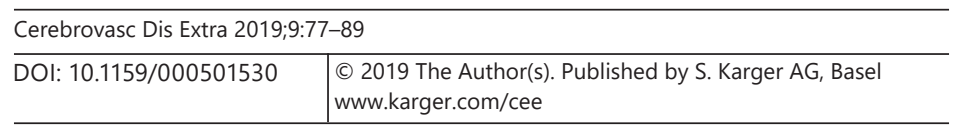

Luong et al.: Effectiveness of Combined EVD with IVF for the Treatment of IVH with $\mathrm{AOH}$

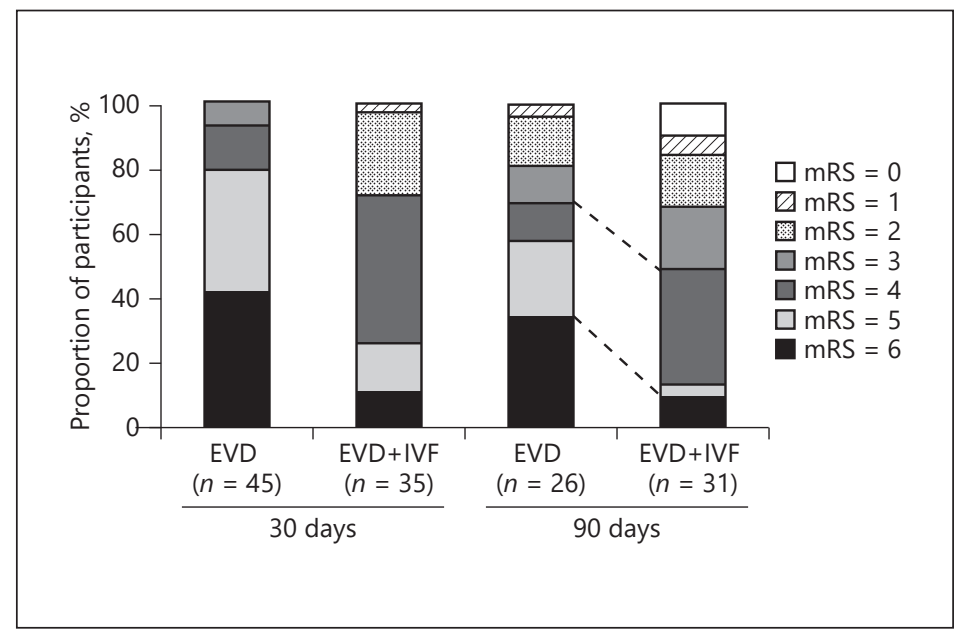

\section{Discussion}

Our prospective cohort study demonstrated that combining EVD with IVF improved the 30- and 90-day mortality rates in IVH patients with AOH (Table 2). A considerable number of studies have assessed the effect of combined EVD with IVF treatment for IVH compared to treatment with EVD alone or EVD plus intraventricular saline in a large patient cohort at various time points. Almost all of the eligible studies were included in three meta-analyses, in which combined EVD with IVF for IVH treatment was reported to be safe, reduce mortality, and potentially lead to an increased number of good functional outcomes. Our findings were in line with the results of these meta-analyses in terms of mortality [13-15]. In addition, the results from our study showed that the number of $1 \mathrm{mg} /$ $\mathrm{mL}$ alteplase doses was significantly associated with mortality (Table 3 ). The recently published CLEAR III trial also showed a significant relationship between clot removal (depending on the number and location of extraventricular drains and the number of alteplase doses) and both mRS score $\leq 3$ and case fatality [4]. Thus, our finding was in line with the results of the CLEAR III trial regarding a significant relationship between the number of alteplase doses and mortality. Our study also showed that "discharged to die" decisions within the first 3 days of care were only made in the EVD group (Table 2), in which almost all patients were in grave condition or dying. The differences in these decisions between the two groups mirrored the fact that patients surviving the initial days after IVH onset had a higher chance to be selected for IVF. However, our further results showed that $94.3 \%(33 / 35)$ of the patients in the EVD+IVF group were assigned immediately before receiving fibrinolysis and within $36 \mathrm{~h}$ of ictus (online suppl. Table A2). Therefore, survivor bias in our study, although possible, is less likely to significantly impact mortality, as suggested by the lower mortality in the EVD+IVF group among patients surviving at least 2 days from admission.

Further analyses showed that our secondary outcome measure, which was a 30-day good functional outcome (mRS score 0-3), was significantly higher in the EVD+IVF group than in the EVD group (Table 2). Our findings seem to be in line with the results of a recently published meta-analysis, which showed that combined EVD with IVF treatment potentially led to an increased number of good functional outcomes [15]. Although no difference was observed in the 90-day good functional outcome (mRS score $0-3$ ) between the two groups (Table 2), there was a trend for a higher rate of 90-day good functional outcome (mRS score 
0-3) in the EVD+IVF group. However, EVD+IVF also increased the risk for patients to be alive with a severe disability (mRS score 4-5) (Fig. 4; online suppl. Table A14). These findings were in line with the results of the CLEAR III trial in terms of functional outcomes [4]. In addition, examination of the relationship between the change in the Graeb score and the functional outcome (30-day mRS score) revealed that less IVH clot removal might result in poorer functional outcome (online suppl. Table A20), and the ventricular blood was removed faster in the EVD+IVF group than in the EVD group (Fig. 3). Therefore, these findings could explain why the outcomes of our study were better than those of the CLEAR III trial in terms of good functional outcomes. However, our findings also revealed that 51.4 and $32.3 \%$ of patients with good functional outcomes, as defined by a GOS score of 3-5, in the EVD and EVD+IVF groups, respectively, had a severe disability (GOS score $=3$, i.e., the patient depends upon others for daily support due to mental or physical disability or both) on day 90 (online suppl. Table A16). Therefore, a GOS score of 3-5 may overestimate the number of patients with a good functional outcome, while an mRS score of 0-3 may yield a more conservative estimate [15].

All patients in our study underwent EVD before assignment, but the EVD duration was significantly shorter in the EVD group than that in the EVD+IVF group (Table 2). However, no significant difference in EVD duration was found among the patients who survived at 30 days between the two groups (Table 2). This result could be explained by the fact that the 10-day case fatality was higher in the EVD group than in the EVD+IVF group (Fig. 2).

Table 2 shows that the proportion of patients with chronic hydrocephalus was significantly lower in the EVD group than in the EVD+IVF group. This result could be biased: the 30-day CT/MRI scan was performed less frequently in the EVD group (Table 2), which could have underestimated the rate of hydrocephalus in this group. However, it is also possible that IVF increased the risk of chronic hydrocephalus. It has been suggested that IVF increases the release of blood products in the CSF, thus increasing the inflammatory process in the arachnoid and fibrosis of the arachnoid granulation. However, in the CLEAR III trial, the rate of ventriculoperitoneal shunt insertion was similar among the two groups [4].

In our study, the complications were distributed evenly across treatment groups (Table 2). However, the proportion of patients with non-neurological infections was higher in our study than in the CLEAR III trial [4], which could be explained in part by the high burden of hospital-acquired infections in hospitals in Vietnam [16, 17].

\section{Limitations of the Study}

This study has several limitations. The primary limitation when assessing any treatment effect from a non-randomized study is the risk of confounding by indication (i.e., the choice to treat with fibrinolysis was tied to the final outcomes in a non-causal manner) and survivor bias (i.e., patients surviving the initial days after ICH had more time to be selected for IVF, causing a spurious association of IVF with survival) [18]. Moreover, our sample size was too small to draw conclusions dealing with functional outcomes. Another limitation was related to the availability and accessibility of rehabilitation services in Vietnam [19-21], which could have significantly impacted the long-term outcomes of our patients.

\section{Conclusions}

In our study, IVF with alteplase was associated with lower mortality in patients with severe IVH requiring EVD. IVF also allowed faster removal of ventricular clots and improved the chance of having a good functional outcome at 1 month. However, there was no benefit regarding long-term functional outcomes. 


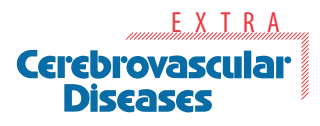

\section{Acknowledgements}

We thank the staff of the Department of Science Management and the Epidemiology Department at Thai Binh University of Medicine and Pharmacy for their support and statistical advice. We also thank all staff members of the Emergency Department and the Radiology Department at Bach Mai Hospital for their support with this study, as well as the Department of Emergency and Critical Care Medicine at Hanoi Medical University. Finally, we thank Dr. Bui Duc Nguyen from the Department of Health and Human Services, the US Centers for Disease Control and Prevention, and the staff of the American Journal Experts for their support with the final version of our manuscript.

\section{Statement of Ethics}

The Research Ethics Committee of Hanoi Medical University (approval document number: 972/QĐ-ĐHYHN) and the Bach Mai Hospital (REC number: BM-2015-80) approved this study. The study was conducted according to the principles of the Declaration of Helsinki. Oral or written informed consent was obtained from the patients or their legally authorized representatives for their anonymized information to be published in this paper.

\section{Disclosure Statement}

The authors declare that there are no potential conflicts of interest with respect to the research, authorship, and/or publication of this article.

\section{Funding Sources}

This research received no specific grant from any funding agency in the public, commercial, or not-for-profit sectors.

\section{Author Contributions}

Chinh Quoc Luong, Anh Dat Nguyen, and Chi Van Nguyen researched the literature and conceived the study. Chinh Quoc Luong, Anh Dat Nguyen, Chi Van Nguyen, Son Ngoc Do, Thu Hong Be, and Lieu Van Nguyen were involved in protocol development and gaining ethical approval. Chinh Quoc Luong, Ton Duy Mai, Phuong Viet Dao, Hung Manh Ngo, Quan Huu Nguyen, Dat Tuan Nguyen, Thong Huu Tran, Ky Van Le, Nam Trong Do, Ngoc Duc Ngo, Vinh Duc Nguyen, Hung Duc Ngo, Hai Bui Hoang, Ha Viet Vu, Lan Tuong Vu, Dai Quoc Khuong, Dung Tien Nguyen, and Trung Xuan Vuong were involved in protocol development and patient recruitment. Dung Thi Pham, Hanh Thi My Pham, Binh Thanh Ngo, and Bai Xuan Nguyen were involved in protocol development and supervised data collection and analysis. Chinh Quoc Luong and Thomas Gaberel wrote the first draft of the manuscript. All authors reviewed and edited the manuscript and approved its final version. Guarantor: Chinh Quoc Luong. 


\section{References}

1 Hanley DF. Intraventricular hemorrhage: severity factor and treatment target in spontaneous intracerebral hemorrhage. Stroke. 2009 Apr;40(4):1533-8.

2 Nishikawa T, Ueba T, Kajiwara M, Miyamatsu N, Yamashita K. A priority treatment of the intraventricular hemorrhage (IVH) should be performed in the patients suffering intracerebral hemorrhage with large IVH. Clin Neurol Neurosurg. 2009 Jun;111(5):450-3.

3 Naff N, Williams MA, Keyl PM, Tuhrim S, Bullock MR, Mayer SA, et al. Low-dose recombinant tissue-type plasminogen activator enhances clot resolution in brain hemorrhage: the intraventricular hemorrhage thrombolysis trial. Stroke. 2011 Nov; 42(11):3009-16.

4 Hanley DF, Lane K, McBee N, Ziai W, Tuhrim S, Lees KR, et al.; CLEAR III Investigators. Thrombolytic removal of intraventricular haemorrhage in treatment of severe stroke: results of the randomised, multicentre, multiregion, placebo-controlled CLEAR III trial. Lancet. 2017 Feb;389(10069):603-11.

5 Morgenstern LB, Hemphill JC 3rd, Anderson C, Becker K, Broderick JP, Connolly ES Jr, et al.; American Heart Association Stroke Council and Council on Cardiovascular Nursing. Guidelines for the management of spontaneous intracerebral hemorrhage: a guideline for healthcare professionals from the American Heart Association/American Stroke Association. Stroke. 2010 Sep;41(9):2108-29.

6 Graeb DA, Robertson WD, Lapointe JS, Nugent RA, Harrison PB. Computed tomographic diagnosis of intraventricular hemorrhage. Etiology and prognosis. Radiology. 1982 Apr;143(1):91-6.

7 Rankin J. Cerebral vascular accidents in patients over the age of 60. II. Prognosis. Scott Med J. 1957 May;2(5): 200-15.

8 Bonita R, Beaglehole R. Recovery of motor function after stroke. Stroke. 1988 Dec;19(12):1497-500.

9 van Swieten JC, Koudstaal PJ, Visser MC, Schouten HJ, van Gijn J. Interobserver agreement for the assessment of handicap in stroke patients. Stroke. 1988 May;19(5):604-7.

10 Jennett B, Bond M. Assessment of outcome after severe brain damage. Lancet. 1975 Mar;1(7905):480-4.

11 Holloway KL, Barnes T, Choi S, Bullock R, Marshall LF, Eisenberg HM, et al. Ventriculostomy infections: the effect of monitoring duration and catheter exchange in 584 patients. J Neurosurg. 1996 Sep;85(3):419-24.

12 Weissleder R, Wittenberg J, Harisinghani MG, Chen JW, Jones SE, Patti JW. Primer of Diagnostic Imaging, 4th Edition. Philadelphia, PA: Mosby Elsevier; 2006.

13 Gaberel T, Magheru C, Parienti JJ, Huttner HB, Vivien D, Emery E. Intraventricular fibrinolysis versus external ventricular drainage alone in intraventricular hemorrhage: a meta-analysis. Stroke. 2011 Oct;42(10):277681.

14 Khan NR, Tsivgoulis G, Lee SL, Jones GM, Green CS, Katsanos AH, et al. Fibrinolysis for intraventricular hemorrhage: an updated meta-analysis and systematic review of the literature. Stroke. 2014 Sep;45(9):2662-9.

15 Baker AD, Rivera Perla KM, Yu Z, Dlugash R, Avadhani R, Mould WA, et al. Fibrinolytic for treatment of intraventricular hemorrhage: a meta-analysis and systematic review. Int J Stroke. 2018 Jan;13(1):11-23.

16 Phu VD, Wertheim HF, Larsson M, Nadjm B, Dinh QD, Nilsson LE, et al. Burden of Hospital Acquired Infections and Antimicrobial Use in Vietnamese Adult Intensive Care Units. PLoS One. 2016 Jan;11(1):e0147544.

17 Phu VD, Nadjm B, Duy NH, Co DX, Mai NT, Trinh DT, et al. Ventilator-associated respiratory infection in a resource-restricted setting: impact and etiology. J Intensive Care. 2017 Dec;5(1):69.

18 Psaty BM, Koepsell TD, Lin D, Weiss NS, Siscovick DS, Rosendaal FR, et al. Assessment and control for confounding by indication in observational studies. J Am Geriatr Soc. 1999 Jun;47(6):749-54.

19 Tirschwell DL, Ton TG, Ly KA, Van Ngo Q, Vo TT, Pham CH, et al. A prospective cohort study of stroke characteristics, care, and mortality in a hospital stroke registry in Vietnam. BMC Neurol. 2012 Dec;12(1):150.

20 Takashima K, Wada K, Tra TT, Smith DR. A review of Vietnam's healthcare reform through the Direction of Healthcare Activities (DOHA). Environ Health Prev Med. 2017 Oct;22(1):74.

21 Hayashi Y, Hai HH, Tai NA. Assessment of the needs of caregivers of stroke patients at state-owned acute-care hospitals in southern Vietnam, 2011. Prev Chronic Dis. 2013 Aug;10:E139. 